\title{
The Acute Effects of Vinyasa Flow Yoga on Arterial Stiffness
}

\author{
ALEXANDER A. PIÑA, ADETOLA FADEYI, JAMES SHADIOW, ANABEL B. \\ CHAVEZ, and STACY D. HUNTER
}

Cardiovascular Physiology Laboratory; Department of Health and Human Performance; Texas State University; San Marcos, TX

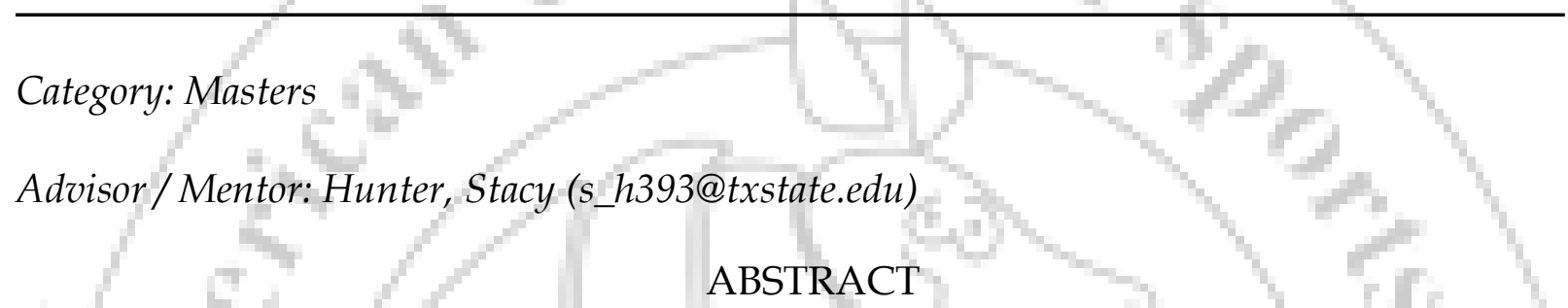

Arterial stiffness (AS) is a marker of subclinical atherosclerotic disease associated with reductions in the buffering capacity of the central, elastic arteries. Previous research has demonstrated reductions in AS with a relatively short-duration, 8-week Bikram (hot) yoga practice, however the acute effects of yoga of any kind on this measure have not been investigated. PURPOSE: The aim of this study was to investigate the acute impact of one bout of Vinyasa flow yoga performed in thermoneutral conditions on indices of AS in healthy adults. METHODS: 20 apparently healthy adults ages 20-75 yrs with at least 3 months of yoga experience completed a one-hour Vinyasa flow yoga DVD. Seated blood pressure measures were obtained pre- and post-intervention. Augmentation index (AIx) and carotid-femoral pulse wave velocity (cfPWV) were measured before and after the yoga session via applanation tonometry. AIx recordings included crude AIx, AIx at a heart rate of 75 beats per minute (AIx@75), and AIx (P2/P1). As associations between negative mood states and impaired endothelial function, a determinant of AS, mood affect was assessed via PANAS 20-item survey before and after the Vinyasa session as well. RESULTS: After completion of the yoga DVD, significant reductions in AIx, AIx@75, and AIx (P2/P1) $(\mathrm{P}<0.05$ for all) were observed. CfPWV ( $\mathrm{P}=0.770$ ) was unaltered. No significant changes in positive mood affect were observed; however negative mood affect significantly decreased $(\mathrm{P}<0.05)$. CONCLUSION: These results highlight the efficacy of a single bout of hatha yoga in improving central and peripheral arterial stiffness measures and provide insight into the potential effects of yoga in mediating CVD risk. These vascular changes were accompanied by significant reductions in negative affect, which could have contributed to reductions in AS via enhanced endothelium-dependent vasodilation.

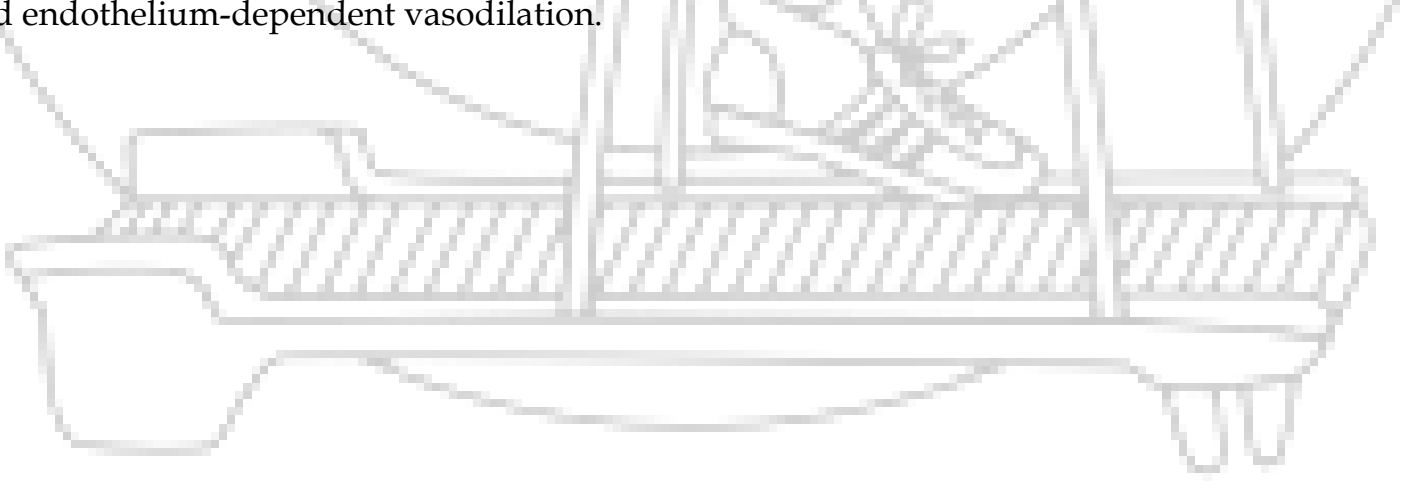

